\title{
Look around your target: a new approach to early diagnosis of lung cancer
}

\author{
Vito R. Papapietro ${ }^{1}$, Gianluca Milanese ${ }^{1}$, Andrea Borghesi ${ }^{2}$, Nicola Sverzellati $^{1}$, Mario Silva $^{1}$ \\ ${ }^{1}$ Section of Radiology, Department of Medicine and Surgery, University of Parma, Parma, Italy; ${ }^{2}$ Department of Radiology, University and Spedali \\ Civili of Brescia, Brescia, Italy \\ Correspondence to: Mario Silva, MD, PhD. Section of Radiology, Unit of Surgical Sciences, Department of Medicine and Surgery (DiMeC), University \\ of Parma, Pad. Barbieri, via Gramsci 14, 43126 Parma, Italy. Email: mario.silva@unipr.it. \\ Comment on: Huang P, Park S, Yan R, et al. Added Value of Computer-aided CT Image Features for Early Lung Cancer Diagnosis with Small \\ Pulmonary Nodules: A Matched Case-Control Study. Radiology 2018;286:286-95.
}

Submitted Oct 18, 2018. Accepted for publication Oct 22, 2018.

doi: $10.21037 / \mathrm{atm} .2018 .10 .56$

View this article at: http://dx.doi.org/10.21037/atm.2018.10.56

Lung cancer is the first oncologic cause of death worldwide (1). Late onset of symptoms and, thus, late diagnosis is one determinant of poor survival. Risk factors are known for selection of asymptomatic subjects at higher risk of lung cancer (2). Secondary prevention by early diagnosis was proved effective by screening trials based on computed tomography (CT) in heavy smokers $(3,4)$, yet with the major limitation of unprecedented high number of nodules detected (5) for which accurate characterization is mandatory with the aim of reducing false positives and associated relevant drawbacks (6-9).

A lung nodule is an extremely frequent finding, mostly with benign histology, therefore it oftentimes becomes a diagnostic clinical challenge. In particular, morbidity and mortality from additional radiographic or unnecessary diagnostic procedures were reported during the work up of small benign lesions or extremely slow-growing neoplasms $(10,11)$. A handful of guidelines drive the management of lung nodules in clinical practice or lung cancer screening (12-15). All guidelines refer to nodule size (either diameter or volume) and more morphologic features (e.g., density, shape, margins, etc.) by visual assessment, which are quite prone to variability (both inter-observer and intraobserver) (12). Therefore, the positive predictive value (PPV) for a small nodule representing cancer can vary according to several subjective determinants, even among thoracic radiologists. In this context, the automatic objective quantification method proposed by Huang raises particular interest (16). Huang and colleagues realized a matched case-control study based on National Lung Screening Trial
(NLST) data to test whether a computer aided diagnosis (CAD) algorithm could increase PPV and reduce FP rate, as compared with evaluation by thoracic radiologists. The authors evaluated features from intranodular, perinodular (parenchyma surrounding the nodule), and extranodular areas and the combination of target, local and global features was tested for overall prediction, in comparison with thoracic radiologist performance. Segmentation was performed by semi-automated technique, which was proven to be more stable and accurate than manual segmentation. Furthermore, semi-automated segmentation was suggested for improvement of the workflow within a lung cancer screening setting, notably by reducing inter-observer differences during analysis of lesions (13).

Among 26,722 NLST screenees, the authors selected individuals displaying a dominant nodule of relatively small size, namely within the range of 4-20 mm diameter. CT images were randomly divided into a training set (for algorithm development) and a validation set: nodules were stratified according to the CAD-derived metric probability of malignancy score $(\mathrm{Pm})$, outperforming visual assignment of risk by the lung-RADS. On the one hand, the semiautomated classification outpointed high malignancy risk in a nodule categorized probably benign by visual reading, the final diagnosis was advanced acinar adenocarcinoma. On the other hand, the semi-automated algorithm did exclude malignancy for a nodule deemed suspicious by visual reading, the final evidence of benignity was draw on the benign biologic behaviour throughout the follow-up period. These representative cases show the potential of Pm score 
as a clinically powerful metric, and thus this preliminary figure encourages thorough investigation towards consistent validation for potential implementation in the setting of lung cancer screening by LDCT, or even clinical practice.

The combination of different demographic data and morphological quantitative CT parameters into a prediction models is especially handy when a summary cancer like score is formulated that includes multifactorial calculation of risk $(8,17)$. In the experiment from Huang et al., CAD increased PPV by 0.43 and decreased FP rate by 0.88 , as compared with the prospective NLST results. Among features, the authors point out a complex quantitative CT parameter (the so-called cp score), which was even a proposed for histologic differentiation, in particular: nodules with highest median cp scores were found to be small-cell carcinoma, papillary adenocarcinoma and squamous cell carcinoma, while nodules with low median cp score were mixed subtypes of adenocarcinoma or nonmucinous adenocarcinoma in situ. This ambitious task appears as revolutionary as insufficient in the current landscape of genomic characterization. It is currently accepted that tumor biopsy is warranted for derivation of several subtypes within the same histologic classification and longitudinal testing of their eventual genomic adaptation through different mechanisms of therapy resistance (18).

The study of Huang et al. falls into the arena of several radiomic methods for stratification of nodule malignancy likelihood. The pioneer studies introduced texture analysis of the quantitative features from within the nodule for stratification of malignancy, this method was further developed for prognostication and prediction of therapy response in lung cancer. For instance, Ravanelli et al. (19) demonstrated that texture features in advanced lung adenocarcinoma provide an independent predictive indicator of response to first-line chemotherapy. Similarly, Ganeshan et al. (20) showed tumour heterogeneity assessed by texture analysis may provide an independent predictor for survival of patients with non-small cell lung cancer (NSCLC). The consistency of quantitative features is restlessly pursued with the aim of granting convenient clinical applicability. In this field, Ciompi et al. (14) proposed a deep learning system capable of automatic processing heterogeneous CT datasets to identify different types of nodules (i.e., solid, non-solid, part-solid, calcified, perifissural and spiculated), also addressing lung-RADS categories. The deep learning method was chosen by Ciompi for its superior ability in classifying nodular lesions, as compared with machine learning approaches. The automatic classification was further specifically developed for identification of benign polygonal perifissural small nodules, namely intraparenchymal lymph nodes, with initial glimpse at the features surrounding the nodule (21). Similarly, vessel involvement assessed by texture analysis was reported as a predictor of cancer risk (22). Vessel involvement as a predictor of lung cancer was evaluated by Liu et al. in screenees of the NLST with negative baseline $\mathrm{CT}$ and lung cancer diagnosis at following rounds and vessel involvement yielded high area under the curve (AUC) for presence of lung cancer. Such feature was confirmed by Huang et al., notably as the second most important parameter to lung cancer likelihood stratification. The assessment of spatial interaction between a small nodule and a vessel introduces the most interesting part of Huang's investigation: the perinodular and extranodular tissue.

The algorithm from Huang and colleagues incorporated perinodular and extranodular features for the definition of the overall likelihood of malignancy. It is unknown whether this algorithm depicts pulmonary abnormalities determined by the tumor growth or parenchymal features which anticipate/favour the cancerogenic process. Nevertheless, this "environmental" radiologic approach to lung cancer is a remarkable complementary point of view, which offers more speculation on the early phases of lung cancer. The same perspective was recently reported in a case series from Borghesi and co-workers, who investigated the outline of tumor surface in small non-solid nodules. They outlined an apparent quantitative cluster capable of discerning between stable and evolving lung nodules, which seems promising for prediction of nodule progression (23). Again, whether this is a cause or a consequence of the neoplastic process is still unknown, yet this characterization appears as relevant as the direct quantification of the intranodular features.

Although encouraging evidences emerge from literature about quantitative CT analysis, some issues still need to be addressed. Indeed, radiomic features may be redundant and non-reproducible, strongly dependent from image acquisition (e.g., tube current, noise index) and reconstruction parameters as well as voxel size- and gray-level discretization-dependent, although different strategies to reduce the weight of these limitations have been extensively tested (24-26). Therefore, it is the natural development of any semi-automated algorithm to be tested on large external multicentre populations before clinical application. This is the near future of the model proposed by Huang et al., since its discovery and validation were operated on a relatively small population and limited to the 
visually determined dominant nodule. Who knows what the machine would have selected as dominant nodule?

In conclusion, the presented article shows a direction within the wide landscape potential of radiomic, notably this technological development is quite sound for optimization of human reading in lung cancer screening. Should this be the beginning of a new era of background artificial intelligence for pre-selection of high-risk CT scan? This perspective should be investigated in the foreseen shortage of human medical professionals, notably radiologists.

\section{Acknowledgements}

None.

\section{Footnote}

Conflicts of Interest: The authors have no conflicts of interest to declare.

\section{References}

1. World Health Organization. 2018. Available online: http:// www.who.int/en/news-room/fact-sheets/detail/the-top10-causes-of-death

2. Kauczor HU, Bonomo L, Gaga M, et al. ESR/ERS white paper on lung cancer screening. Eur Respir J 2015;46:28-39.

3. Aberle DR, Adams AM, Berg CD, et al. Reduced lungcancer mortality with low-dose computed tomographic screening. N Engl J Med 2011;365:395-409.

4. Infante M, Sestini S, Galeone C, et al. Lung cancer screening with low-dose spiral computed tomography: evidence from a pooled analysis of two Italian randomized trials. Eur J Cancer Prev 2017;26:324-9.

5. Silva M, Pastorino U, Sverzellati N. Lung cancer screening with low-dose CT in Europe: strength and weakness of diverse independent screening trials. Clin Radiol 2017;72:389-400.

6. Henschke CI, Yip R, Yankelevitz DF, et al. Definition of a positive test result in computed tomography screening for lung cancer: a cohort study. Ann Intern Med 2013;158:246-52.

7. Yip R, Henschke CI, Yankelevitz DF, et al. CT Screening for Lung Cancer: Alternative Definitions of Positive Test Result Based on the National Lung Screening Trial and International Early Lung Cancer Action Program Databases. Radiology 2014;273:591-6.
8. Horeweg N, van Rosmalen J, Heuvelmans MA, et al. Lung cancer probability in patients with CT-detected pulmonary nodules: a prespecified analysis of data from the NELSON trial of low-dose CT screening. Lancet Oncol 2014;15:1332-41.

9. Horeweg N, Scholten ET, de Jong PA, et al. Detection of lung cancer through low-dose CT screening (NELSON): a prespecified analysis of screening test performance and interval cancers. Lancet Oncol 2014;15:1342-50.

10. Bach PB, Mirkin JN, Oliver TK, et al. Benefits and harms of CT screening for lung cancer: a systematic review. JAMA 2012;307:2418-29.

11. Silva M, Prokop M, Jacobs C, et al. Long-Term Active Surveillance of Screening Detected Subsolid Nodules is a Safe Strategy to Reduce Overtreatment. J Thorac Oncol 2018;13:1454-63.

12. MacMahon H, Naidich DP, Goo JM, et al. Guidelines for Management of Incidental Pulmonary Nodules Detected on CT Images: From the Fleischner Society 2017. Radiology 2017;284:228-43.

13. Lung-RADS Version 1.0 Assessment Categories: American College of Radiology; 2014. Available online: http://www. acr.org/ /media/ACR/Documents/PDF/QualitySafety/ Resources/LungRADS/AssessmentCategories

14. Ciompi F, Chung K, van Riel SJ, et al. Towards automatic pulmonary nodule management in lung cancer screening with deep learning. Sci Rep 2017;7:46479.

15. Oudkerk M, Devaraj A, Vliegenthart R, et al. European position statement on lung cancer screening. Lancet Oncol 2017;18:e754-66.

16. Huang P, Park S, Yan R, et al. Added Value of Computeraided CT Image Features for Early Lung Cancer Diagnosis with Small Pulmonary Nodules: A Matched Case-Control Study. Radiology 2018;286:286-95.

17. McWilliams A, Tammemagi MC, Mayo JR, et al. Probability of cancer in pulmonary nodules detected on first screening CT. N Engl J Med 2013;369:910-9.

18. Recondo G, Facchinetti F, Olaussen KA, et al. Making the first move in EGFR-driven or ALK-driven NSCLC: firstgeneration or next-generation TKI? Nat Rev Clin Oncol 2018;15:694-708.

19. Ravanelli M, Farina D, Morassi M, et al. Texture analysis of advanced non-small cell lung cancer (NSCLC) on contrast-enhanced computed tomography: prediction of the response to the first-line chemotherapy. Eur Radiol 2013;23:3450-5.

20. Ganeshan B, Panayiotou E, Burnand K, et al. Tumour heterogeneity in non-small cell lung carcinoma assessed 


\section{Page 4 of 4}

by CT texture analysis: a potential marker of survival. Eur Radiol 2012;22:796-802.

21. Schreuder A, van Ginneken B, Scholten ET, et al. Classification of CT Pulmonary Opacities as Perifissural Nodules: Reader Variability. Radiology 2018;288:867-75.

22. Liu Y, Wang H, Li Q, et al. Radiologic Features of Small Pulmonary Nodules and Lung Cancer Risk in the National Lung Screening Trial: A Nested Case-Control Study. Radiology 2018;286:298-306.

23. Borghesi A, Michelini S, Bertagna F, et al. Hilly or mountainous surface: a new CT feature to predict the behavior of pure ground glass nodules? Eur J Radiol Open

Cite this article as: Papapietro VR, Milanese G, Borghesi A, Sverzellati N, Silva M. Look around your target: a new approach to early diagnosis of lung cancer. Ann Transl Med 2018;6(Suppl 1):S77. doi: 10.21037/atm.2018.10.56

\section{Papapietro et al. Automatic stratification of early lung cancer}

2018;5:177-82.

24. Berenguer R, Pastor-Juan MD, Canales-Vazquez J, et al. Radiomics of CT Features May Be Nonreproducible and Redundant: Influence of CT Acquisition Parameters. Radiology 2018;288:407-15.

25. Mackin D, Fave X, Zhang L, et al. Measuring Computed Tomography Scanner Variability of Radiomics Features. Invest Radiol 2015;50:757-65.

26. Silva M, Milanese G, Seletti V, et al. Pulmonary quantitative CT imaging in focal and diffuse disease: current research and clinical applications. Br J Radiol 2018;91:20170644. 